\title{
STRUCTURAL COMPOSITION AND DISTRIBUTION OF TREE SPECIES OF DUDHPUKURIA-DHOPACHORI WILDLIFE SANCTUARY, CHITTAGONG, BANGLADESH
}

\author{
Hossain, M. A., M. K. Hossain, M. S. Alam and M. M. Abdullah-Al-Mamun \\ Institute of Forestry and Environmental Sciences, University of Chittagong, Chittagong-4331, \\ Bangladesh
}

\begin{abstract}
Structural composition based on diameter and height class distribution of the tree species of DudhpukuriaDhopachori Wildlife Sanctuary, Chittagong, Bangladesh was assessed. A total of 183 tree species having $\geq 10$ DBH belonging to 125 genera and 48 families was recorded from the study area. Different species were found to dominate in different DBH classes, i.e. Aporosa wallichii (4.06\%), Artocarpus chama (1.54\%) and Dillenia pentagyna $(0.38 \%)$ dominated $10-<25 \mathrm{~cm}, 25-<40 \mathrm{~cm}$ and $40-<55 \mathrm{~cm}$ DBH, respectively. Swintonia floribunda was found in maximum (7 out of 8 ) number of DBH classes. Both the percentage of tree individuals $(73.18 \%)$ and number of species (169) were highest in $10-24.5 \mathrm{~cm}$ DBH range. Similarly, Dipterocarpus turbinatus $(14.5-24.4 \mathrm{~m}$ and $24.5-34.4 \mathrm{~m})$ and Swintonia floribunda $(34.5-44.4 \mathrm{~m}$ and $44.5-54.4 \mathrm{~m})$ were dominant in two height ranges each. On the other hand, height range $4.5-14.4 \mathrm{~m}$ was dominated by Aporosa wallichii (4.36\%). Dipterocarpus costatus, Artocarpus chama, Syzygium firmum and Bombax insigne were found in all the height classes. Both the number of tree species and number of individual stems (167 species; 1,684 individuals) were highest in the height range of $4.5-14.4 \mathrm{~m}$. The number of species and tree individuals decreased linearly with increasing height. All these indicate that, there was a sustainable natural regeneration and successful recruitment of Aporosa wallichii, Dipterocarpus turbinatus, Lithocarpus acuminata, Grewia nervosa and Artocarpus chama in Dudhpukuria-Dhopachori Wildlife Sanctuary. Dipterocarpus turbinatus was found as dominant species as it constitures maximum (5.56\%) percentage of all tree individuals. The findings of the study may be useful for protection, conservation and sustainable management of Dudhpukuria-Dhopachori Wildlife Sanctuary.
\end{abstract}

Keywords: Dudhpukuria-Dhopachori, DBH and height class, Conservation, Dipterocarpus turbinatus.

\section{INTRODUCTION}

Bangladesh covers an area of 1.204 million ha of natural forests which are classified into hill forests, Sal forests and mangrove forests of the Sundarbans (Altrell et al. 2007). The flora of hill forests of Bangladesh is closely related to Indo-China than any other forests of this region (Das and Alam 2001). An undisturbed natural hill forest of Bangladesh is generally uneven-aged and multi-storied forest (Alam 2008). Clear felling followed by artificial regeneration in the hill forests of Bangladesh caused serious harm to natural regeneration, seedling and sapling establishment, soil fertility, natural forest condition and hence the natural ecosystem (Das 1980, Haque and Alam 1988). In Bangladesh, forest biological resources are severely disturbed and degraded due to rapid population growth, poverty, inappropriate forest management system, over exploitation, energy deficit and lack of motivation regarding biodiversity conservation (Hassan 1995). The overall forest structure is changed by such disturbances (Shaforth et al. 2002) which ultimately affects the regeneration and population dynamics (Kwit and Platt 2003). Diameter distribution of trees has been often used to represent forest structure (Khan et al. 1987, Newton and Smith 1988). A forest community typically includes a canopy, an understory layer, shrubs and herbs to ground layer, and the forest floor, including roots and soil. The vertical arrangement of vegetation in a forest is as important to many species as the size of the forest itself. It is suggested that vertical stratification of vegetation results in the stratification of food 
resources and microclimate, animal communities and each vegetation stratum having its own characteristic fauna (Kimmins 1996).

Knowledge about the tree flora of a country or an area is very important for its sustainable use and future management plan. The trees as key structural components of forest ecosystems, provide timber and non-timber products, and vital ecosystem services. Various research findings have suggested that tree species diversity can be used as a surrogate for overall species diversity in forest ecosystems (SBSTTA 1996, Lammerts van Bueren and Duivenvoorden 1996). Tree diversity is often considered as a good proxy to estimate diversity of other taxonomic groups (Gentry 1988). A clear understanding of forest stands parameters, i.e. DBH class and height class distribution are also important for modeling future wood production of that forests. Motaleb and Hossain (2009) and Nath et al. (1997) investigated suitable management system based on diameter class distribution in two natural forests of Bangladesh. Dudhpukuria-Dhopachori declared Wildlife Sanctuary in 2010 and is composed of disturbed and undulated natural forest patches. Hence, this study is conducted to find out the species composition, height and diameter distribution at a class interval of $10 \mathrm{~m}$ and $15 \mathrm{~cm}$, respectively in DudhpukuriaDhopachori Wildlife Sanctuary, so that management decision for the forest can be facilitated.

\section{MATERIAL AND METHODS}

The study was carried out in the Dudhpukuria-Dhopachori Wildlife Sanctuary (between $22^{\circ} 09^{\prime}$ and $22^{\circ} 22^{\prime}$ north latitude and $92^{\circ} 05^{\prime}$ and $92^{\circ} 10^{\prime}$ east longitudes) along the borderline of Chittagong, Rangamati and Bandarban districts (Hossain et al. 2014). The Wildlife Sanctuary (WS) comprises a portion of the Reserved Forests of Khurusia and Dohazari Forest Ranges under Chittagong (South) Forest Division. The sanctuary comprises with a total area of 4,716.57 ha. The whole wildlife sanctuary area, criss-crossed by numerous creeks, is comprised of hill and hillocks (about $80 \%$ of total area) and plain lands (about $20 \%$ of total area) covered with forests and grasses. Stratified Random Sample method was applied for the inventory of the tree species. A total of 125 sample plots of $20 \mathrm{~m} \times 20 \mathrm{~m}$ in size were taken from the three blocks (Dudhpukuria- 31 plots, Kamalachori- 31 plots and Dhopachori63 plots) randomly to cover a total sample area of 5 ha for the assessment of the tree species diversity. From each quadrat, all the tree species having DBH of $\geq 10 \mathrm{~cm}$ were recorded by their name and number. Tree individuals of each species were tallied into DBH classes at $15 \mathrm{~cm}$ interval and height classes at 10 $\mathrm{m}$ interval. Herbarium specimens were collected and preserved following standard scientific methods. The specimens were identified with the help of taxonomists and comparing with the voucher specimen of the Bangladesh Forest Research Institute and Chittagong University herbaria.

\section{RESULTS AND DISCUSSION}

The results revealed that Dudhpukuria-Dhopachori Wildlife Sanctuary harbors 183 tree species belonging to 125 genera and 48 families. The number of tree stems per hectare is 468 (Table 1).

\section{Diameter $(D B H)$ class distribution of tree species}

Percentage distribution of tree individuals in different DBH classes $(\mathrm{cm})$ shows that $73.18 \%$ trees belong to DBH class ranges from $10-<25 \mathrm{~cm}$. On the other hand, DBH range $100-<115 \mathrm{~cm}$ was found to represent lowest percentage $(0.17 \%)$ of trees. Different DBH classes were dominated by different tree species, i.e. DBH range $10-<25 \mathrm{~cm}$ was dominated by Aporosa wallichii $(4.06 \%)$ followed by Lithocarpus acuminata (3.76\%) and Dipterocarpus turbinatus (3.55\%); DBH range $25-<40 \mathrm{~cm}$ was dominated by Artocarpus chama (1.54\%) followed by Dipterocarpus turbinatus (1.45\%) and Lithocarpus acuminata (0.86\%). Moreover, Dillenia pentagyna (0.38\%), Dipterocarpus turbinatus 
(0.26\%), Terminalia bellirica $(0.09 \%)$ and Dipterocarpus costatus $(0.09 \%)$ were dominant in 40 $<55 \mathrm{~cm}, 55-<70 \mathrm{~cm}, 85-<100 \mathrm{~cm}$ and $100-<115 \mathrm{~cm}$ DBH ranges, respectively. Swintonia floribunda occurred in higher DBH classes $70-<85 \mathrm{~cm}$ and $\geq 115 \mathrm{~cm}$ with representation of $0.13 \%$ and $0.09 \%$ respectively (Table 1).

Table 1. Diameter class distribution of tree species of Dudhpukuria-Dhopachori Wildlife Sanctuary.

\begin{tabular}{|c|c|c|c|c|c|c|c|c|c|c|}
\hline \multirow[t]{2}{*}{ No } & \multirow[t]{2}{*}{ Scientific name } & \multicolumn{9}{|c|}{ Percentage distribution of tree species into different diameter classes $(\mathrm{cm})$} \\
\hline & & $10-<25$ & $25-<40$ & $40-<55$ & $55-<70$ & $70-<85$ & $85-<100$ & $100-<115$ & $\geq 115$ & Total $(\%)$ \\
\hline 1 & Acacia auriculiformis & 0.09 & - & - & - & - & - & - & - & 0.09 \\
\hline 2 & Acacia mangium & 0.09 & - & - & - & - & - & - & - & 0.09 \\
\hline 3 & Acronychia pedunculata & 1.92 & 0.3 & 0.04 & - & - & - & - & - & 2.27 \\
\hline 4 & Actinodaphne angustifolia & 0.64 & 0.09 & - & - & - & - & - & - & 0.73 \\
\hline 5 & Aegle marmelos & 0.09 & - & - & - & - & - & - & - & 0.09 \\
\hline 6 & Aglaia chittagonga & 0.3 & 0.09 & - & - & - & - & - & - & 0.38 \\
\hline 7 & Aglaia perviridis & 0.17 & 0.13 & - & - & - & - & - & - & 0.3 \\
\hline 8 & Aglaia spectabilis & 0.04 & - & 0.04 & - & - & 0.04 & - & - & 0.13 \\
\hline 9 & Aidia oppositifolia & 0.17 & 0.04 & - & - & - & - & - & - & 0.21 \\
\hline 10 & Alangium chinense & 0.47 & 0.21 & 0.09 & - & - & - & - & - & 0.77 \\
\hline 11 & Albizia chinensis & 0.94 & 0.09 & - & - & - & - & - & - & 1.03 \\
\hline 12 & Albizia odoratissima & 0.68 & 0.17 & 0.09 & - & - & - & - & - & 0.94 \\
\hline 13 & Albizia procera & 0.26 & 0.13 & - & 0.04 & - & - & - & - & 0.43 \\
\hline 14 & Alstonia scholaris & 0.3 & - & - & - & - & - & - & - & 0.3 \\
\hline 15 & Anisoptera scaphula & 0.09 & 0.04 & - & - & - & - & - & - & 0.13 \\
\hline 16 & Anogeissus acuminata & 0.81 & 0.17 & - & - & - & - & - & - & 0.98 \\
\hline 17 & Antidesma banius & 0.04 & - & - & - & - & - & - & - & 0.04 \\
\hline 18 & Antidesma velutinum & 0.09 & - & - & - & - & - & - & - & 0.09 \\
\hline 19 & Aphanamixis polystachya & 0.56 & 0.21 & 0.04 & - & - & - & - & - & 0.81 \\
\hline 20 & Aporosa wallichii & 4.06 & 0.34 & 0.04 & 0.04 & 0.04 & - & - & - & 4.53 \\
\hline 21 & Aquilaria agallocha & 0.09 & - & - & - & - & - & - & - & 0.09 \\
\hline 22 & Artocarpous heterophyllus & 0.09 & 0.04 & - & - & - & - & - & - & 0.13 \\
\hline 23 & Artocarpus chama & 2.14 & 1.54 & 0.04 & 0.04 & - & - & - & - & 3.76 \\
\hline 24 & Artocarpus lacucha & 0.68 & 0.17 & 0.09 & - & - & - & - & - & 0.94 \\
\hline 25 & Baccaurea ramiflora & 0.04 & - & - & - & - & - & - & - & 0.04 \\
\hline 26 & Berrya cordifolia & 0.09 & 0.04 & 0.17 & 0.04 & - & - & - & - & 0.34 \\
\hline 27 & Bombax insigne & 0.21 & 0.21 & 0.09 & - & - & - & - & - & 0.51 \\
\hline 28 & Brassaiopsis glomerulata & 0.04 & - & - & - & - & - & - & - & 0.04 \\
\hline 29 & Bridelia retusa & 0.13 & - & - & - & - & - & - & - & 0.13 \\
\hline 30 & Brownlowia elata & 0.13 & 0.04 & - & - & - & - & - & - & 0.17 \\
\hline 31 & Butea monosperma & 0.04 & - & - & - & - & - & - & - & 0.04 \\
\hline 32 & Caesalpinia pulcherrima & - & 0.04 & - & - & - & - & - & - & 0.04 \\
\hline 33 & Callicarpa arborea & 1.24 & 0.21 & - & - & - & - & - & - & 1.45 \\
\hline 34 & Calophyllum polyanthum & 0.04 & 0.04 & 0.04 & - & - & - & - & - & 0.13 \\
\hline 35 & Canthium horridum & 0.09 & - & - & - & - & - & - & - & 0.09 \\
\hline 36 & Carallia brachiata & 0.21 & - & 0.04 & - & 0.04 & - & - & - & 0.3 \\
\hline 37 & Caryota mitis & 0.51 & 0.04 & - & - & - & - & - & - & 0.56 \\
\hline 38 & Caryota urens & 0.26 & - & - & - & - & - & - & - & 0.26 \\
\hline 39 & Cassia fistula & 0.13 & 0.09 & - & - & - & - & - & - & 0.21 \\
\hline 40 & Cassia nodosa & 0.04 & - & - & - & - & - & - & - & 0.04 \\
\hline 41 & Ceriscoides campanulata & 0.09 & - & - & - & - & - & - & - & 0.09 \\
\hline 42 & Chisocheton cumingianus & 0.47 & 0.21 & 0.04 & 0.09 & 0.09 & - & - & - & 0.9 \\
\hline 43 & Chukrasia tabularis & 0.26 & 0.56 & 0.26 & - & - & - & - & - & 1.07 \\
\hline 44 & Cinnamoтит iners & 0.13 & - & - & - & - & - & - & - & 0.13 \\
\hline 45 & Cocos nucifera & 0.09 & 0.04 & - & - & - & - & - & - & 0.13 \\
\hline
\end{tabular}




\begin{tabular}{|c|c|c|c|c|c|c|c|c|c|c|}
\hline 46 & Crypteronia paniculata & 0.04 & 0.04 & - & - & - & - & - & - & 0.09 \\
\hline 47 & Cryptocarya amygdalina & 0.26 & 0.34 & 0.13 & - & - & - & - & - & 0.73 \\
\hline 48 & Derris robusta & - & 0.04 & - & - & - & - & - & - & 0.04 \\
\hline 49 & Didymosperma gracilis & 0.09 & - & - & - & - & - & - & - & 0.09 \\
\hline 50 & Dillenia indica & 0.04 & - & - & - & - & - & - & - & 0.04 \\
\hline 51 & Dillenia pentagyna & 0.26 & 0.47 & 0.38 & 0.13 & - & - & - & - & 1.24 \\
\hline 52 & Diospyros malabarica & 0.17 & 0.04 & 0.04 & - & 0.04 & - & - & - & 0.3 \\
\hline 53 & Diospyros toposia & - & - & 0.04 & - & - & - & - & - & 0.04 \\
\hline 54 & Dipterocarpus alatus & 0.04 & 0.09 & - & 0.04 & - & - & 0.04 & 0.04 & 0.26 \\
\hline 55 & Dipterocarpus costatus & 0.51 & 0.09 & - & 0.09 & 0.09 & - & 0.09 & 0.09 & 0.94 \\
\hline 56 & Dipterocarpus turbinatus & 3.55 & 1.45 & 0.17 & 0.26 & 0.09 & 0.04 & - & - & 5.56 \\
\hline 57 & Discospermum abnorme & 0.04 & - & - & - & - & - & - & - & 0.04 \\
\hline 58 & Drimycarpus racemosus & 0.09 & 0.04 & - & - & - & - & - & - & 0.13 \\
\hline 59 & Duabanga grandiflora & 0.09 & 0.13 & - & - & - & - & - & - & 0.21 \\
\hline 60 & Dysoxylum binectariferum & 0.04 & 0.04 & - & - & - & - & - & - & 0.09 \\
\hline 61 & Dysoxylum excelsum & 0.09 & - & - & - & - & - & - & - & 0.09 \\
\hline 62 & Ehretias errata & - & 0.04 & - & - & - & - & - & - & 0.04 \\
\hline 63 & Elaeocarpus floribundus & 0.17 & 0.13 & - & 0.04 & 0.04 & - & - & - & 0.38 \\
\hline 64 & Elaeocarpus tectorius & 0.34 & 0.04 & 0.04 & - & 0.04 & - & - & - & 0.47 \\
\hline 65 & Elaeocarpus varunиa & 0.04 & 0.04 & - & - & - & - & - & - & 0.09 \\
\hline 66 & Engelhardtia spicata & - & 0.04 & - & - & - & - & - & - & 0.04 \\
\hline 67 & Erythrina fusca & 0.17 & 0.09 & - & 0.04 & - & - & - & - & 0.3 \\
\hline 68 & Ficus auriculata & 0.13 & 0.04 & - & - & - & - & - & - & 0.17 \\
\hline 69 & Ficus benghalensis & 0.04 & 0.04 & - & 0.04 & 0.04 & 0.04 & - & 0.04 & 0.26 \\
\hline 70 & Ficus fistulosa & 0.04 & - & - & - & - & - & - & - & 0.04 \\
\hline 71 & Ficus hispida & 0.9 & 0.09 & - & - & - & - & - & - & 0.98 \\
\hline 72 & Ficus lamponga & 0.04 & 0.09 & 0.09 & 0.09 & - & 0.04 & 0.04 & - & 0.38 \\
\hline 73 & Ficus nervosa & 0.13 & - & 0.09 & 0.13 & 0.09 & - & - & - & 0.43 \\
\hline 74 & Ficus racemosa & 0.38 & 0.04 & 0.09 & - & - & - & - & - & 0.51 \\
\hline 75 & Ficus semicordata & 0.17 & - & - & - & - & - & - & - & 0.17 \\
\hline 76 & Ficus variegata & 0.9 & 0.04 & 0.09 & 0.04 & 0.04 & - & - & - & 1.11 \\
\hline 77 & Flacourtia jangomas & 0.04 & - & - & - & - & - & - & - & 0.04 \\
\hline 78 & Garcinia cowa & 0.68 & 0.43 & - & - & - & - & - & - & 1.11 \\
\hline 79 & Garcinia morella & 0.09 & 0.04 & - & - & - & - & - & - & 0.13 \\
\hline 80 & Garcinia xanthochymus & 0.09 & 0.09 & 0.04 & - & - & - & - & - & 0.21 \\
\hline 81 & Gardenia coronaria & 0.04 & - & - & - & - & - & - & - & 0.04 \\
\hline 82 & Garuga pinnata & 0.9 & 0.21 & 0.26 & 0.09 & 0.04 & - & - & - & 1.5 \\
\hline 83 & Glochidion lanceolarium & 0.04 & - & - & - & - & - & - & - & 0.04 \\
\hline 84 & Glochidion multiloculare & 0.86 & - & - & - & - & - & - & - & 0.86 \\
\hline 85 & Glochidion velutinum & 0.09 & - & - & - & - & - & - & - & 0.09 \\
\hline 86 & Gluta elegans & 0.34 & 0.26 & 0.04 & 0.09 & - & - & - & - & 0.73 \\
\hline 87 & Glycosmis pentaphylla & 0.04 & - & - & - & - & - & - & - & 0.04 \\
\hline 88 & Gmelina arborea & 0.3 & 0.09 & - & - & - & - & - & - & 0.38 \\
\hline 89 & Grevillea robusta & - & - & - & - & 0.04 & - & - & - & 0.04 \\
\hline 90 & Grewia nervosa & 3.42 & 0.6 & 0.04 & 0.04 & - & - & - & - & 4.11 \\
\hline 91 & Grewia tiliifolia & 0.13 & - & - & - & - & - & - & - & 0.13 \\
\hline 92 & Haldina cordifolia & 0.13 & - & - & - & - & - & - & - & 0.13 \\
\hline 93 & Harpullia cupanoides & - & 0.04 & - & - & - & - & - & - & 0.04 \\
\hline 94 & Holarrhena antidysenterica & 0.86 & - & - & - & - & - & - & - & 0.86 \\
\hline 95 & Hopea odorata & 0.13 & - & - & - & - & - & - & - & 0.13 \\
\hline 96 & Hydnocarpus laurifolius & 0.9 & 0.21 & - & - & - & - & - & - & 1.11 \\
\hline 97 & Illex godajam & 0.09 & - & - & - & - & - & - & - & 0.09 \\
\hline 98 & Lagerstroemia macrocarpa & 0.56 & - & - & - & - & - & - & - & 0.56 \\
\hline 99 & Lagerstromia speciosa & 0.26 & 0.13 & - & - & - & - & - & - & 0.38 \\
\hline
\end{tabular}




\begin{tabular}{|c|c|c|c|c|c|c|c|c|c|c|}
\hline 100 & Lannea coromandelica & 0.04 & - & - & - & - & - & - & - & 0.04 \\
\hline 101 & Leea robusta & - & 0.04 & - & - & - & - & - & - & 0.04 \\
\hline 102 & Lepisanthes rubiginosa & 0.04 & - & - & - & - & - & - & - & 0.04 \\
\hline 103 & Licuala peltata & 0.64 & 0.17 & 0.04 & - & - & - & - & 0.04 & 0.9 \\
\hline 104 & Lithocarpus acuminata & 3.76 & 0.86 & 0.04 & - & - & - & - & - & 4.66 \\
\hline 105 & Lithocarpus elegans & 0.3 & 0.17 & - & - & - & - & - & - & 0.47 \\
\hline 106 & Lithocarpus pachyphylla & 0.56 & 0.13 & 0.21 & 0.04 & - & - & - & - & 0.94 \\
\hline 107 & Lithocarpus polystachya & 0.68 & 0.17 & - & - & - & - & - & - & 0.86 \\
\hline 108 & Litsea glutinosa & 0.47 & - & - & - & - & - & - & - & 0.47 \\
\hline 109 & Macaranga denticulata & 1.03 & 0.17 & - & - & - & - & - & - & 1.2 \\
\hline 110 & Macaranga indica & 0.13 & - & - & - & - & - & - & - & 0.13 \\
\hline 111 & Macaranga peltata & 0.09 & - & - & - & - & - & - & - & 0.09 \\
\hline 112 & Maesa chisia & 0.04 & - & - & - & - & - & - & - & 0.04 \\
\hline 113 & Maesa indica & 0.43 & 0.04 & - & - & - & - & - & - & 0.47 \\
\hline 114 & Magifera sylvatica & 0.13 & 0.09 & - & - & 0.04 & - & - & - & 0.26 \\
\hline 115 & Mallotus tetracoccus & 0.04 & - & - & - & - & - & - & - & 0.04 \\
\hline 116 & Mangifera indica & - & 0.13 & - & - & - & - & - & - & 0.13 \\
\hline 117 & Michelia bailloni & - & 0.04 & - & - & - & - & - & - & 0.04 \\
\hline 118 & Micromelum minutum & 0.17 & - & - & - & - & - & - & - & 0.17 \\
\hline 119 & Mitragyna diversifolia & 0.81 & 0.04 & - & - & - & - & - & - & 0.86 \\
\hline 120 & Mitragyna parvifolia & 1.07 & 0.04 & - & - & - & - & - & - & 1.11 \\
\hline 121 & Mitragyna rotundifolia & 0.26 & - & - & - & - & - & - & - & 0.26 \\
\hline 122 & Myristica linifolia & 0.26 & - & - & - & - & - & - & - & 0.26 \\
\hline 123 & Neolamarckia cadamba & 0.68 & - & - & - & - & - & - & - & 0.68 \\
\hline 124 & Neonauclea sessilifolia & 0.13 & - & - & - & - & - & - & - & 0.13 \\
\hline 125 & Ormosia robusta & 0.04 & - & - & - & - & - & - & - & 0.04 \\
\hline 126 & Oroxylum indicum & 0.38 & - & - & - & - & - & - & - & 0.38 \\
\hline 127 & Pajanelia longifolia & 0.09 & - & - & - & - & - & - & - & 0.09 \\
\hline 128 & Palaquium polyanthum & 0.09 & - & 0.09 & - & - & - & - & - & 0.17 \\
\hline 129 & Persea bombycina & 0.04 & - & - & - & - & - & - & - & 0.04 \\
\hline 130 & Phoebe lanceolata & 0.04 & - & - & - & - & - & - & - & 0.04 \\
\hline 131 & Phoenix sylvestris & 0.09 & 0.04 & - & - & - & - & - & - & 0.13 \\
\hline 132 & Phyllanthus emblica & 0.38 & 0.04 & - & - & - & - & - & - & 0.43 \\
\hline 133 & Phyllanthus reticulatus & 0.04 & - & - & - & - & - & - & - & 0.04 \\
\hline 134 & Picrasma javanica & 0.09 & - & - & - & - & - & - & - & 0.09 \\
\hline 135 & Pithecellobium angulatum & 0.21 & 0.26 & 0.04 & - & - & - & - & - & 0.51 \\
\hline 136 & Protium serratum & 2.01 & 0.47 & 0.13 & - & - & - & - & - & 2.61 \\
\hline 137 & Psidium guajaba & 0.04 & - & - & - & - & - & - & - & 0.04 \\
\hline 138 & Pterospermum acerifolium & 0.68 & 0.04 & - & - & - & - & - & - & 0.73 \\
\hline 139 & Pterospermum semisagittatum & 0.77 & 0.13 & 0.09 & - & - & - & - & - & 0.98 \\
\hline 140 & Sapium baccatum & 0.26 & 0.13 & 0.17 & 0.04 & 0.04 & - & - & - & 0.64 \\
\hline 141 & Saprosma ternatum & 0.04 & - & - & - & - & - & - & - & 0.04 \\
\hline 142 & Sarcochlamys pulcherrima & 0.09 & - & - & - & - & - & - & - & 0.09 \\
\hline 143 & Schima wallichii & 1.58 & 0.51 & 0.04 & 0.17 & 0.09 & - & - & - & 2.4 \\
\hline 144 & Senna siamea & 0.3 & 0.04 & - & 0.04 & - & - & - & - & 0.38 \\
\hline 145 & Shorea robusta & - & 0.04 & - & - & - & - & - & - & 0.04 \\
\hline 146 & Siphonodon celastrineus & 0.3 & 0.04 & - & - & - & - & - & - & 0.34 \\
\hline 147 & Spondias pinnata & 0.64 & 0.09 & - & - & - & - & - & - & 0.73 \\
\hline 148 & Sterculia foetida & 0.6 & 0.09 & 0.09 & - & - & - & - & - & 0.77 \\
\hline 149 & Sterculia hamiltonii & 0.04 & - & - & - & - & - & - & - & 0.04 \\
\hline 150 & Sterculia villosa & 0.38 & 0.3 & - & - & - & - & - & - & 0.68 \\
\hline 151 & Stereospermum colais & 1.28 & 0.34 & 0.34 & 0.17 & - & - & - & - & 2.14 \\
\hline 152 & Stereospermum suaveolens & 0.38 & 0.04 & - & 0.04 & - & - & - & - & 0.47 \\
\hline 153 & Streblus asper & 0.21 & 0.21 & - & - & - & - & - & - & 0.43 \\
\hline
\end{tabular}




\begin{tabular}{|c|c|c|c|c|c|c|c|c|c|c|}
\hline 154 & Suregada multiflora & 0.04 & - & - & - & - & - & - & - & 0.04 \\
\hline 155 & Swietenia mahagoni & - & 0.04 & - & - & - & - & - & - & 0.04 \\
\hline 156 & Swintonia floribunda & 0.04 & 0.04 & 0.09 & 0.09 & 0.13 & 0.04 & - & 0.09 & 0.51 \\
\hline 157 & Syzygium balsameum & 0.13 & 0.04 & - & - & 0.04 & - & - & - & 0.21 \\
\hline 158 & Syzygium claviflorum & - & - & 0.09 & - & - & - & - & - & 0.09 \\
\hline 159 & Syzygium cumini & 0.17 & - & - & - & - & - & - & - & 0.17 \\
\hline 160 & Syzygium cymosum & 0.04 & - & - & - & - & - & - & - & 0.04 \\
\hline 161 & Syzygium firmum & 0.13 & 0.13 & 0.13 & 0.17 & 0.09 & - & - & - & 0.64 \\
\hline 162 & Syzygium fruticosum & 0.17 & 0.04 & - & - & - & - & - & - & 0.21 \\
\hline 163 & Syzygium ramosissimum & 0.04 & - & 0.04 & - & - & - & - & - & 0.09 \\
\hline 164 & Syzygium syzygioides & 0.21 & 0.09 & - & - & - & - & - & - & 0.3 \\
\hline 165 & Syzygium tetragonum & 0.38 & - & - & - & - & - & - & - & 0.38 \\
\hline 166 & Tamarindus indica & 0.13 & - & - & - & - & - & - & - & 0.13 \\
\hline 167 & Tarenna campaniflora & 1.75 & 0.09 & - & - & - & - & - & - & 1.84 \\
\hline 168 & Tectona grandis & 3.08 & 0.13 & 0.04 & 0.04 & - & - & - & 0.04 & 3.34 \\
\hline 169 & Terminalia alata & 0.04 & 0.09 & - & - & - & - & - & - & 0.13 \\
\hline 170 & Terminalia arjuna & 0.26 & 0.04 & 0.13 & 0.09 & - & - & - & - & 0.51 \\
\hline 171 & Terminalia bellirica & 1.54 & 0.13 & 0.3 & 0.17 & 0.04 & 0.09 & - & - & 2.27 \\
\hline 172 & Terminalia chebula & 0.04 & 0.04 & - & 0.04 & - & - & - & - & 0.13 \\
\hline 173 & Tetrameles nudiflora & 0.21 & 0.17 & - & - & - & - & - & - & 0.38 \\
\hline 174 & Toona ciliata & 0.43 & 0.09 & - & - & - & - & - & - & 0.51 \\
\hline 175 & Trema orientalis & 0.04 & - & - & - & - & - & - & - & 0.04 \\
\hline 176 & Trewia nudiflora & 0.3 & 0.17 & - & - & - & - & - & - & 0.47 \\
\hline 177 & Vatica lanceaefolia & - & 0.04 & - & - & - & - & - & - & 0.04 \\
\hline 178 & Vitex glabrata & 0.26 & - & 0.04 & - & - & - & - & - & 0.3 \\
\hline 179 & Vitex peduncularis & 2.31 & - & 0.04 & - & - & - & - & - & 2.35 \\
\hline 180 & Vitex pinnata & 0.43 & 0.09 & 0.04 & - & - & - & - & - & 0.56 \\
\hline 181 & Walsura robusta & 0.09 & 0.04 & - & - & - & - & - & - & 0.13 \\
\hline 182 & Wrightia arborea & 0.09 & 0.04 & - & - & - & - & - & - & 0.13 \\
\hline 183 & Zanthoxylum rhetsa & 0.17 & 0.09 & - & - & - & - & - & - & 0.26 \\
\hline & Total & 73.18 & 17.45 & 4.88 & 2.48 & 1.2 & 0.3 & 0.17 & 0.34 & 100 \\
\hline
\end{tabular}

Occurrences of individual tree species at different diameter classes showed that Swintonia floribunda occurred in 7 DBH classes among 8 classes, but its representation was only $0.51 \%$. Followed by $S$. floribunda, presence of tree individuals of D. turbinatus, Terminalia bellirica, D. costatus, Ficus benghalensis and $F$. lamponga were noticed in maximum 6 DBH classes. It indicates sustainable natural regeneration and successful recruitment of these tree species in the study area. As an individual species, D. turbinatus showed maximum (5.56\%) occurrence of its individuals followed by Lithocarpus acuminata (4.66\%), Aposora wallichii (4.53\%), Grewia nervosa (4.11\%) and Artocarpus chama $(3.76 \%)$. Distribution of individual stems among different DBH classes, shown in Fig. 1, indicates a reverse J-shaped curve which is typical for most undisturbed tropical forests (Campbell et al. 1986; Rankin-de-Merona et al. 1992). It depicts progressive decline of tree individuals in larger tree size (DBH) classes with age.

Similarly, the number of tree species reflects a decreasing trend with increasing diameter (Fig. 1). Both the number of species and number of tree individuals were maximum (169 species; 1,711 individuals) in $10-<25 \mathrm{~cm}$ DBH range and minimum (3 species; 4 individual stems) in $100-<115 \mathrm{DBH}$ range (Fig. 1). The trend reveals successful recruitment of some pioneer tree species. It also indicates incidence of illegal felling of comparatively mature trees in Wildlife Sanctuary area. Recent strengthening of protection over the forests supposed to increase the higher number of trees in lower size classes. 
Height class distribution of tree species

Percentage distribution of tree individuals into different height classes shows that smallest growth class, i.e. $4.5-<14.4 \mathrm{~m}$ height range holds maximum $(72.03 \%)$ tree individuals, whereas the minimum $(0.47 \%)$ tree species was represented by the $44.5-<54.4 \mathrm{~m}$ height range (Table 2). Similar to DBH classes, height classes were dominated by different tree species. Aporosa wallichii (4.36\%) was dominant in 4.5-<14.4m height range followed by Lithocarpus acuminata $(3.68 \%)$ and Grewia nervosa (3.55\%). Height range $14.5-<24.4 \mathrm{~m}$ was dominated by D. turbinatus $(2.22 \%)$ followed by L. acuminata $(0.98 \%)$ and Stereospermum colais $(0.9 \%)$. On the other hand, individuals of D. turbinatus was found maximum $(0.77 \%)$ in $24.5-<34.4 \mathrm{~m}$ height range followed by A. chama $(0.38 \%)$. Swintonia floribunda $(0.17 \%)$ was found dominant in $34.5-<44.4 \mathrm{~m}$ and $44.5-<54.4$ height ranges. The overall distribution reflects that the natural environment for sustainable natural regeneration and successful recruitment of D. turbinatus, L. acuminata, Grewia nervosa and A. chama was suitable in Dudhpukuria-Dhopachori forests. Dipterocarpus costatus, Artocarpus chama, Syzygium firmum and Bombax insigne were found in all the height classes (Table 2).

Table 2. Tree species distribution of Dudhpukuria- Dhopachori Wildlife Sanctuary in different height classes.

\begin{tabular}{|c|c|c|c|c|c|c|c|}
\hline \multirow[t]{2}{*}{ No. } & \multirow[t]{2}{*}{ Scientific name } & \multicolumn{6}{|c|}{ Distribution (percentage) of tree species in different height class } \\
\hline & & $4.5-<14.4$ & $14.5-<24.4$ & $24.5-<34.4$ & $34.5-<44.4$ & $44.5-<54.4$ & Total \\
\hline 1 & Acacia auriculiformis & 0.04 & 0.04 & - & - & - & 0.09 \\
\hline 2 & Acacia mangium & - & 0.09 & - & - & - & 0.09 \\
\hline 3 & Acronychia pedunculata & 1.71 & 0.56 & - & - & - & 2.27 \\
\hline 4 & Actinodaphne angustifolia & 0.64 & 0.09 & - & - & - & 0.73 \\
\hline 5 & Aegle marmelos & 0.09 & - & - & - & - & 0.09 \\
\hline 6 & Aglaia chittagonga & 0.30 & 0.09 & - & - & - & 0.38 \\
\hline 7 & Aglaia perviridis & 0.21 & 0.04 & 0.04 & - & - & 0.30 \\
\hline 8 & Aglaia spectabilis & 0.04 & 0.04 & 0.04 & - & - & 0.13 \\
\hline 9 & Aidia oppositifolia & 0.17 & 0.04 & - & - & - & 0.21 \\
\hline 10 & Alangium chinense & 0.51 & 0.26 & - & - & - & 0.77 \\
\hline 11 & Albizia chinensis & 0.94 & 0.09 & - & - & - & 1.03 \\
\hline 12 & Albizia odoratissima & 0.64 & 0.30 & - & - & - & 0.94 \\
\hline 13 & Albizia procera & 0.17 & 0.21 & 0.04 & - & - & 0.43 \\
\hline 14 & Alstonia scholaris & 0.30 & - & - & - & - & 0.30 \\
\hline 15 & Anisoptera scaphula & 0.09 & 0.09 & - & - & - & 0.17 \\
\hline 16 & Anogeissus acuminata & 0.90 & 0.09 & - & - & - & 0.98 \\
\hline 17 & Antidesma banius & 0.04 & - & - & - & - & 0.04 \\
\hline 18 & Antidesma velutinum & 0.09 & - & - & - & - & 0.09 \\
\hline 19 & Aphanamixis polystachya & 0.64 & 0.17 & - & - & - & 0.81 \\
\hline 20 & Aporosa wallichii & 4.36 & 0.17 & - & - & - & 4.53 \\
\hline 21 & Aquilaria agallocha & 0.09 & - & - & - & - & 0.09 \\
\hline 22 & Artocarpous heterophyllus & 0.13 & - & - & - & - & 0.13 \\
\hline 23 & Artocarpus chama & 2.35 & 0.86 & 0.38 & 0.13 & 0.04 & 3.76 \\
\hline 24 & Artocarpus lacucha & 0.56 & 0.38 & - & - & - & 0.94 \\
\hline 25 & Baccaurea ramiflora & 0.04 & - & - & - & - & 0.04 \\
\hline 26 & Berrya cordifolia & - & 0.09 & 0.26 & - & - & 0.34 \\
\hline 27 & Bombax insigne & 0.21 & 0.13 & 0.09 & 0.04 & 0.04 & 0.51 \\
\hline 28 & Brassaiopsis glomerulata & 0.04 & - & - & - & - & 0.04 \\
\hline 29 & Bridelia retusa & 0.13 & - & - & - & - & 0.13 \\
\hline 30 & Brownlowia elata & 0.13 & 0.04 & - & - & - & 0.17 \\
\hline 31 & Butea monosperma & 0.04 & - & - & - & - & 0.04 \\
\hline 32 & Caesalpinia pulcherrima & 0.04 & - & - & - & - & 0.04 \\
\hline
\end{tabular}




\begin{tabular}{|c|c|c|c|c|c|c|c|}
\hline 33 & Callicarpa arborea & 1.24 & 0.21 & - & - & - & 1.45 \\
\hline 34 & Calophyllum polyanthum & - & 0.09 & - & 0.04 & - & 0.13 \\
\hline 35 & Canthium horridum & 0.09 & - & - & - & - & 0.09 \\
\hline 36 & Carallia brachiata & 0.17 & 0.04 & 0.09 & - & - & 0.30 \\
\hline 37 & Caryota mitis & 0.56 & - & - & - & - & 0.56 \\
\hline 38 & Caryota urens & 0.26 & - & - & - & - & 0.26 \\
\hline 39 & Cassia fistula & 0.17 & 0.04 & - & - & - & 0.21 \\
\hline 40 & Cassia nodosa & 0.04 & - & - & - & - & 0.04 \\
\hline 41 & Ceriscoides campanulata & 0.09 & - & - & - & - & 0.09 \\
\hline 42 & Chisocheton cumingianus & 0.47 & 0.30 & 0.13 & - & - & 0.90 \\
\hline 43 & Chukrasia tabularis & 0.13 & 0.81 & 0.13 & - & - & 1.07 \\
\hline 44 & Cinnamoтит iners & 0.13 & - & - & - & - & 0.13 \\
\hline 45 & Cocos nucifera & 0.04 & 0.09 & - & - & - & 0.13 \\
\hline 46 & Crypteronia paniculata & - & 0.09 & - & - & - & 0.09 \\
\hline 47 & Cryptocarya amygdalina & 0.47 & 0.26 & - & - & - & 0.73 \\
\hline 48 & Derris robusta & 0.04 & - & - & - & - & 0.04 \\
\hline 49 & Didymosperma gracilis & 0.09 & - & - & - & - & 0.09 \\
\hline 50 & Dillenia indica & 0.04 & - & - & - & - & 0.04 \\
\hline 51 & Dillenia pentagyna & 0.68 & 0.43 & 0.13 & - & - & 1.24 \\
\hline 52 & Diospyros malabarica & 0.17 & 0.09 & 0.04 & - & - & 0.30 \\
\hline 53 & Diospyros toposia & - & 0.04 & - & - & - & 0.04 \\
\hline 54 & Dipterocarpus alatus & - & 0.13 & 0.04 & 0.04 & 0.04 & 0.26 \\
\hline 55 & Dipterocarpus costatus & 0.51 & 0.09 & 0.09 & 0.13 & 0.13 & 0.94 \\
\hline 56 & Dipterocarpus turbinatus & 2.48 & 2.22 & 0.77 & 0.09 & - & 5.56 \\
\hline 57 & Discospermum abnorme & 0.04 & - & - & - & - & 0.04 \\
\hline 58 & Drimycarpus racemosus & 0.09 & 0.04 & - & - & - & 0.13 \\
\hline 59 & Duabanga grandiflora & 0.09 & 0.13 & - & - & - & 0.21 \\
\hline 60 & Dysoxylum binectariferum & 0.09 & - & - & - & - & 0.09 \\
\hline 61 & Dysoxylum excelsum & 0.09 & - & - & - & - & 0.09 \\
\hline 62 & Ehretia serrata & - & 0.04 & - & - & - & 0.04 \\
\hline 63 & Elaeocarpus floribundus & 0.13 & 0.26 & - & - & - & 0.38 \\
\hline 64 & Elaeocarpus tectorius & 0.34 & 0.13 & - & - & - & 0.47 \\
\hline 65 & Elaeocarpus vагипиа & - & 0.09 & - & - & - & 0.09 \\
\hline 66 & Engelhardtia spicata & 0.04 & - & - & - & - & 0.04 \\
\hline 67 & Erythrina fusca & 0.17 & 0.13 & - & - & - & 0.30 \\
\hline 68 & Ficus auriculata & 0.17 & - & - & - & - & 0.17 \\
\hline 69 & Ficus benghalensis & 0.09 & 0.09 & 0.09 & - & - & 0.26 \\
\hline 70 & Ficus fistulosa & 0.04 & - & - & - & - & 0.04 \\
\hline 71 & Ficus hispida & 0.94 & 0.04 & - & - & - & 0.98 \\
\hline 72 & Ficus lamponga & 0.17 & 0.21 & - & - & - & 0.38 \\
\hline 73 & Ficus nervosa & 0.13 & 0.04 & 0.21 & 0.04 & - & 0.43 \\
\hline 74 & Ficus racemosa & 0.38 & 0.13 & - & - & - & 0.51 \\
\hline 75 & Ficus semicordata & 0.09 & 0.09 & - & - & - & 0.17 \\
\hline 76 & Ficus variegata & 0.81 & 0.26 & 0.04 & - & - & 1.11 \\
\hline 77 & Flacourtia jangomas & 0.04 & - & - & - & - & 0.04 \\
\hline 78 & Garcinia cowa & 0.94 & 0.17 & - & - & - & 1.11 \\
\hline 79 & Garcinia morella & 0.04 & 0.09 & - & - & - & 0.13 \\
\hline 80 & Garcinia xanthochymus & 0.04 & 0.13 & - & 0.04 & - & 0.21 \\
\hline 81 & Gardenia coronaria & 0.04 & - & - & - & - & 0.04 \\
\hline 82 & Garuga pinnata & 0.81 & 0.51 & 0.17 & - & - & 1.50 \\
\hline 83 & Glochidion lanceolarium & 0.04 & - & - & - & - & 0.04 \\
\hline 84 & Glochidion multiloculare & 0.77 & 0.09 & - & - & - & 0.86 \\
\hline 85 & Glochidion velutinum & 0.09 & - & - & - & - & 0.09 \\
\hline 86 & Gluta elegans & 0.26 & 0.34 & 0.13 & - & - & 0.73 \\
\hline
\end{tabular}




\begin{tabular}{|c|c|c|c|c|c|c|c|}
\hline 87 & Glycosmis pentaphylla & 0.04 & - & - & - & - & 0.04 \\
\hline 88 & Gmelina arborea & 0.38 & - & - & - & - & 0.38 \\
\hline 89 & Grevillea robusta & - & - & 0.04 & - & - & 0.04 \\
\hline 90 & Grewia nervosa & 3.55 & 0.56 & - & - & - & 4.11 \\
\hline 91 & Grewia tiliifolia & 0.13 & - & - & - & - & 0.13 \\
\hline 92 & Haldina cordifolia & 0.13 & - & - & - & - & 0.13 \\
\hline 93 & Harpullia cupanoides & - & 0.04 & - & - & - & 0.04 \\
\hline 94 & Holarrhena antidysenterica & 0.81 & 0.04 & - & - & - & 0.86 \\
\hline 95 & Hopea odorata & 0.13 & - & - & - & - & 0.13 \\
\hline 96 & Hydnocarpus laurifolius & 0.86 & 0.26 & - & - & - & 1.11 \\
\hline 97 & Illex godajam & 0.09 & - & - & - & - & 0.09 \\
\hline 98 & Lagerstroemia macrocarpa & 0.51 & 0.04 & - & - & - & 0.56 \\
\hline 99 & Lagerstromia speciosa & 0.21 & 0.17 & - & - & - & 0.38 \\
\hline 100 & Lannea coromandelica & 0.04 & - & - & - & - & 0.04 \\
\hline 101 & Leea robusta & 0.04 & - & - & - & - & 0.04 \\
\hline 102 & Lepisanthes rubiginosa & 0.04 & - & - & - & - & 0.04 \\
\hline 103 & Licuala peltata & 0.43 & 0.38 & 0.09 & - & - & 0.90 \\
\hline 104 & Lithocarpus acuminata & 3.68 & 0.98 & - & - & - & 4.66 \\
\hline 105 & Lithocarpus elegans & 0.04 & 0.43 & - & - & - & 0.47 \\
\hline 106 & Lithocarpus pachyphylla & 0.64 & 0.26 & 0.04 & - & - & 0.94 \\
\hline 107 & Lithocarpus polystachya & 0.81 & 0.04 & - & - & - & 0.86 \\
\hline 108 & Litsea glutinosa & 0.47 & - & - & - & - & 0.47 \\
\hline 109 & Macaranga denticulata & 1.11 & 0.09 & - & - & - & 1.20 \\
\hline 110 & Macaranga indica & 0.09 & 0.04 & - & - & - & 0.13 \\
\hline 111 & Macaranga peltata & 0.09 & - & - & - & - & 0.09 \\
\hline 112 & Maesa chisia & 0.04 & - & - & - & - & 0.04 \\
\hline 113 & Maesa indica & 0.43 & 0.04 & - & - & - & 0.47 \\
\hline 114 & Mangifera sylvatica & 0.13 & 0.09 & 0.04 & - & - & 0.26 \\
\hline 115 & Mallotus tetracoccus & 0.04 & - & - & - & - & 0.04 \\
\hline 116 & Mangifera indica & 0.04 & 0.09 & - & - & - & 0.13 \\
\hline 117 & Michelia bailloni & - & - & 0.04 & - & - & 0.04 \\
\hline 118 & Micromelum minutum & 0.17 & - & - & - & - & 0.17 \\
\hline 119 & Mitragyna diversifolia & 0.77 & 0.09 & - & - & - & 0.86 \\
\hline 120 & Mitragyna parvifolia & 1.03 & 0.09 & - & - & - & 1.11 \\
\hline 121 & Mitragyna rotundifolia & 0.26 & - & - & - & - & 0.26 \\
\hline 122 & Myristica linifolia & 0.26 & - & - & - & - & 0.26 \\
\hline 123 & Neolamarckia cadamba & 0.60 & 0.09 & - & - & - & 0.68 \\
\hline 124 & Neonauclea sessilifolia & 0.13 & - & - & - & - & 0.13 \\
\hline 125 & Ormosia robusta & 0.04 & - & - & - & - & 0.04 \\
\hline 126 & Oroxylum indicum & 0.38 & - & - & - & - & 0.38 \\
\hline 127 & Pajanelia longifolia & 0.04 & 0.04 & - & - & - & 0.09 \\
\hline 128 & Palaquium polyanthum & 0.09 & 0.04 & - & - & - & 0.13 \\
\hline 129 & Persea bombycina & 0.04 & - & - & - & - & 0.04 \\
\hline 130 & Phoebe lanceolata & 0.04 & - & - & - & - & 0.04 \\
\hline 131 & Phoenix sylvestris & 0.09 & 0.04 & - & - & - & 0.13 \\
\hline 132 & Phyllanthus emblica & 0.43 & - & - & - & - & 0.43 \\
\hline 133 & Phyllanthus reticulatus & 0.04 & - & - & - & - & 0.04 \\
\hline 134 & Picrasma javanica & 0.09 & - & - & - & - & 0.09 \\
\hline 135 & Pithecellobium angulatum & 0.26 & 0.26 & - & - & - & 0.51 \\
\hline 136 & Protium serratum & 2.31 & 0.30 & - & - & - & 2.61 \\
\hline 137 & Psidium guajaba & 0.04 & - & - & - & - & 0.04 \\
\hline 138 & Pterospermum acerifolium & 0.68 & 0.04 & - & - & - & 0.73 \\
\hline 139 & Pterospermum semisagittatum & 0.73 & 0.26 & - & - & - & 0.98 \\
\hline 140 & Sapium baccatum & 0.34 & 0.30 & - & - & - & 0.64 \\
\hline
\end{tabular}




\begin{tabular}{|c|c|c|c|c|c|c|c|}
\hline 141 & Saprosma ternatum & 0.04 & - & - & - & - & 0.04 \\
\hline 142 & Sarcochlamys pulcherrima & 0.09 & - & - & - & - & 0.09 \\
\hline 143 & Schima wallichii & 1.54 & 0.73 & 0.13 & - & - & 2.40 \\
\hline 144 & Senna siamea & 0.26 & 0.13 & - & - & - & 0.38 \\
\hline 145 & Shorea robusta & - & - & - & 0.04 & - & 0.04 \\
\hline 146 & Siphonodon celastrineus & 0.26 & 0.09 & - & - & - & 0.34 \\
\hline 147 & Spondias pinnata & 0.56 & 0.17 & - & - & - & 0.73 \\
\hline 148 & Sterculia foetida & 0.60 & 0.17 & - & - & - & 0.77 \\
\hline 149 & Sterculia hamiltonii & 0.04 & - & - & - & - & 0.04 \\
\hline 150 & Sterculia villosa & 0.43 & 0.26 & - & - & - & 0.68 \\
\hline 151 & Stereospermum colais & 1.07 & 0.90 & 0.17 & - & - & 2.14 \\
\hline 152 & Steteospermum suaveolens & 0.34 & 0.13 & - & - & - & 0.47 \\
\hline 153 & Streblus asper & 0.38 & 0.04 & - & - & - & 0.43 \\
\hline 154 & Suregada multiflora & - & 0.04 & - & - & - & 0.04 \\
\hline 155 & Swietenia mahagoni & 0.04 & - & - & - & - & 0.04 \\
\hline 156 & Swintonia floribunda & 0.04 & - & 0.13 & 0.17 & 0.17 & 0.51 \\
\hline 157 & Syzygium balsameum & 0.09 & 0.13 & - & - & - & 0.21 \\
\hline 158 & Syzygium claviflorum & - & - & 0.09 & - & - & 0.09 \\
\hline 159 & Syzygium cumini & 0.13 & 0.04 & - & - & - & 0.17 \\
\hline 160 & Syzygium cymosum & - & 0.04 & - & - & - & 0.04 \\
\hline 161 & Syzygium firmum & 0.09 & 0.21 & 0.21 & 0.13 & - & 0.64 \\
\hline 162 & Syzygium fruticosum & 0.17 & 0.04 & - & - & - & 0.21 \\
\hline 163 & Syzygium ramosissimum & 0.04 & - & 0.04 & - & - & 0.09 \\
\hline 164 & Syzygium syzygioides & 0.21 & 0.09 & - & - & - & 0.30 \\
\hline 165 & Syzygium tetragonum & 0.34 & 0.04 & - & - & - & 0.38 \\
\hline 166 & Tamarindus indica & 0.09 & 0.04 & - & - & - & 0.13 \\
\hline 167 & Tarenna campaniflora & 1.67 & 0.17 & - & - & - & 1.84 \\
\hline 168 & Tectona grandis & 3.17 & 0.17 & - & - & - & 3.34 \\
\hline 169 & Terminalia alata & 0.04 & 0.09 & - & - & - & 0.13 \\
\hline 170 & Terminalia arjuna & 0.26 & 0.26 & - & - & - & 0.51 \\
\hline 171 & Terminalia bellirica & 1.37 & 0.81 & 0.04 & 0.04 & - & 2.27 \\
\hline 172 & Terminalia chebula & 0.09 & 0.04 & - & - & - & 0.13 \\
\hline 173 & Tetrameles nudiflora & 0.17 & 0.17 & 0.04 & - & - & 0.38 \\
\hline 174 & Toona ciliata & 0.38 & 0.13 & - & - & - & 0.51 \\
\hline 175 & Trema orientalis & 0.04 & - & - & - & - & 0.04 \\
\hline 176 & Trewia nudiflora & 0.30 & 0.17 & - & - & - & 0.47 \\
\hline 177 & Vatica lanceaefolia & - & - & 0.04 & - & - & 0.04 \\
\hline 178 & Vitex glabrata & 0.26 & 0.04 & - & - & - & 0.30 \\
\hline 179 & Vitex peduncularis & 2.22 & 0.13 & - & - & - & 2.35 \\
\hline 180 & Vitex pinnata & 0.47 & 0.09 & - & - & - & 0.56 \\
\hline 181 & Walsura robusta & 0.13 & - & - & - & - & 0.13 \\
\hline 182 & Wrightia arborea & 0.09 & 0.04 & - & - & - & 0.13 \\
\hline 183 & Zanthoxylum rhetsa & 0.17 & 0.09 & - & - & - & 0.26 \\
\hline \multicolumn{2}{|c|}{$\begin{array}{c}\text { Total } \\
\end{array}$} & 72.03 & 22.54 & 4.06 & 0.94 & 0.43 & 100.00 \\
\hline
\end{tabular}

Distribution of tree individuals among different height classes depicted a reverse J-shaped curve which indicates presence of stable population structure or good regeneration status as of a typical natural forest. Patterns of height $(\mathrm{m})$ class distribution designate general trends of population dynamics and recruitment process to the maximum species. The number of species and individuals decreased regularly with increasing total height (Fig. 2). These were also highest (167 species; 1,684 individuals) in the height range of $4.5-<14.4 \mathrm{~m}$ whereas $44.5-<54.4 \mathrm{~m}$ height range showed lowest number of tree species and individuals (Fig. 2). 


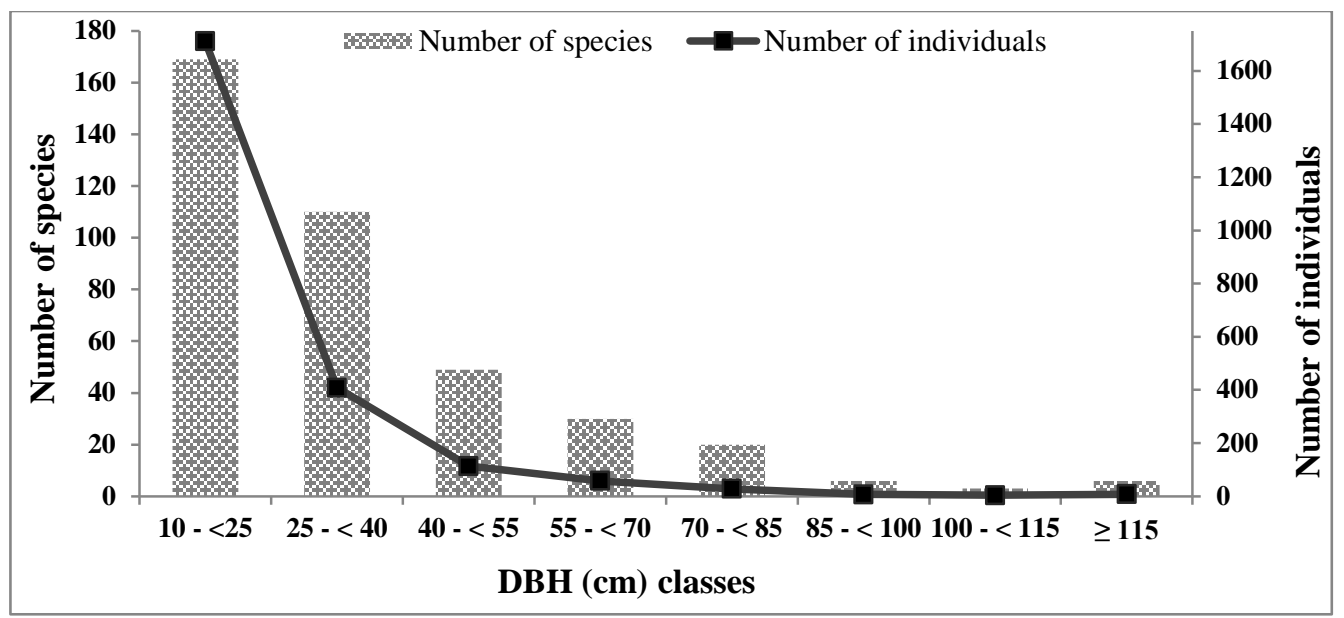

Fig. 1. Distribution of tree species and individuals in different DBH $(\mathrm{cm})$ classes of Dudhpukuria-Dhopachori Wildlife Sanctuary.

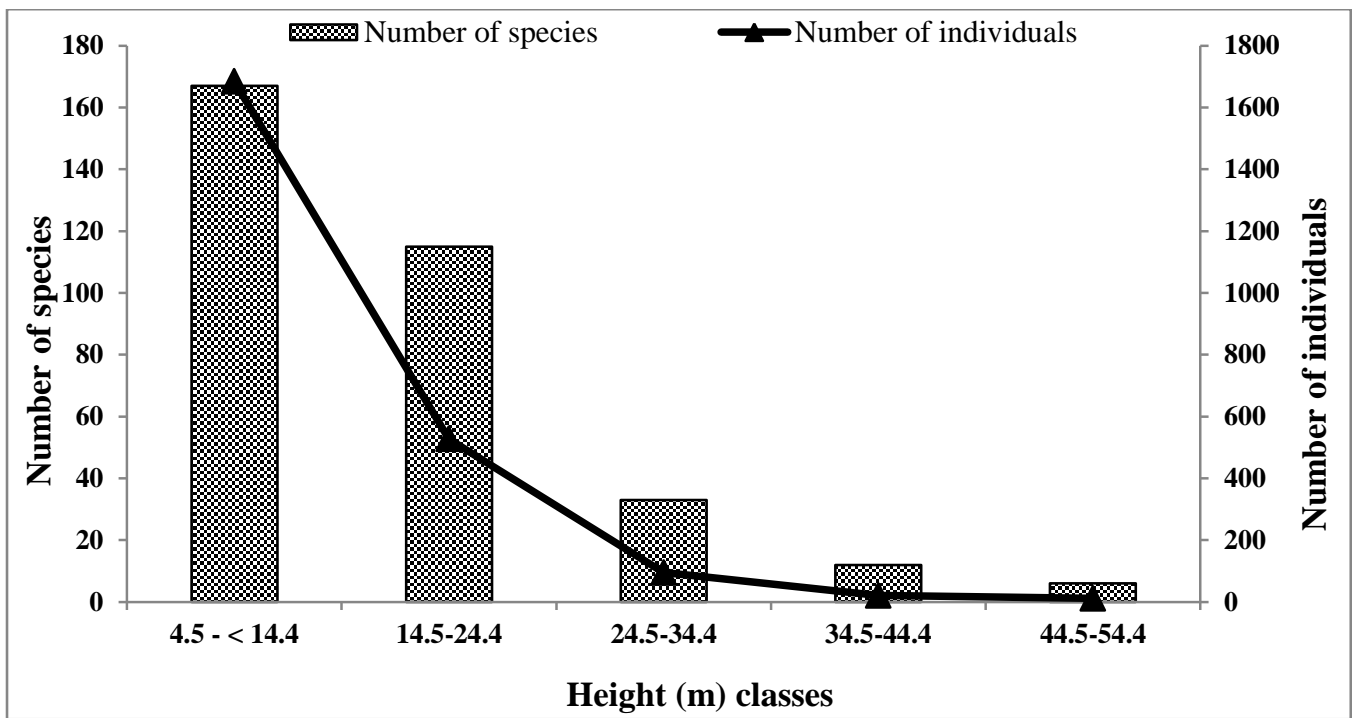

Fig. 2. Distribution of tree species and individuals in different height (m) classes of Dudhpukuria-Dhopachori Wildlife Sanctuary.

\section{Height - Diameter Relationship}

Both the tree height and diameter at breast height (DBH) show that maximum number of species and individual stems are decreasing with height and diameter growth (Fig. 3). The typical distribution of tree species of Dudhpukuria-Dhopachori Wildlife Sanctuary is a bit different in comparison to tropical forests. The numbers of mature tree individuals are decreasing because of illegal felling, firewood collection and occurrence of incendiary fire during dry season. Silvicultural treatments for stand improvement are crucial for further growth and development of each species. Quantitative characteristics of trees are crucial for forest management planning. Predicting stand structure and overall status in diameter or height class distribution is of great interest to forest managers for predicting the evaluation of forest resources and scheduling the future silvicultural treatments (Nanos and Montero 2002). As revealed in the results, there is an unevenness of age of the trees in the DudhpukuriaDhopachori Wildlife Sanctuary. Trees were distributed in different size classes. The unevenness of age and structure is made by nature. So, estimation of tree distribution in diameter classes can be used as a 
model (Sheykholeslami et al. 2011) to manage similar other stands of uneven-aged high forests of Bangladesh. Height $(\mathrm{m})$ and DBH $(\mathrm{cm})$ relationship $\left(\mathrm{r}^{2}=0.603\right)$ indicates that silvicultural treatments are essential for the appropriate growth and development of the tree species of the forest.

The earlier research findings of similar studies reported that maximum number of species (51 species) and tree stem individuals (200 individuals) were found to occur within DBH range of $20-<30$ $\mathrm{cm}$ and $10-<20 \mathrm{~cm}$, respectively in Sitapahar Reserve Forest, Chittagong, Bangladesh (Nath et al. 1997). The authors also observed that different species dominate different DBH classes and $D$. turbinatus consisting $16.77 \%$ of all individuals was most dominant species. Motaleb and Hossain (2009) found that $\mathrm{DBH}$ range $20-<30 \mathrm{~cm}$ possess both maximum number of species (58 species) and maximum (465 stems /ha) tree individuals in Tankawati Natural Forest Reserve of Chittagong (South) Forest Division, Bangladesh. The highest percentage $(29.69 \%)$ of trees was also occupied by $20-<30 \mathrm{~cm}$ DBH class in this forest along with 1,305 stems per hectare. Ahmed and Haque (1993) reported number of stems (257 stems/ha) showing highest (30.53\%) presence in 30-39cm DBH class and lowest (1.32\%) in $80-89 \mathrm{~cm}$ DBH range from the natural forests of Bangladesh. In comparison to the above studies, Dudhpukuria-Dhopachori Wildlife Sanctuary possesses a typical trend of population structure, distribution and recruitment processes of the species. Some species have higher number of individuals in the first DBH classes $(10-<25 \mathrm{~cm})$ demonstrates their potential regeneration of potentials. Other species possess either no or poor number of individuals in the lowest DBH classes suggesting that they have poor regeneration status and should be prioritized for conservation. Regular diameter distribution should be maintained by enhancing natural regeneration and protection from the stand point of wood utilization, species diversity and maximizing the multiple functions of forest.

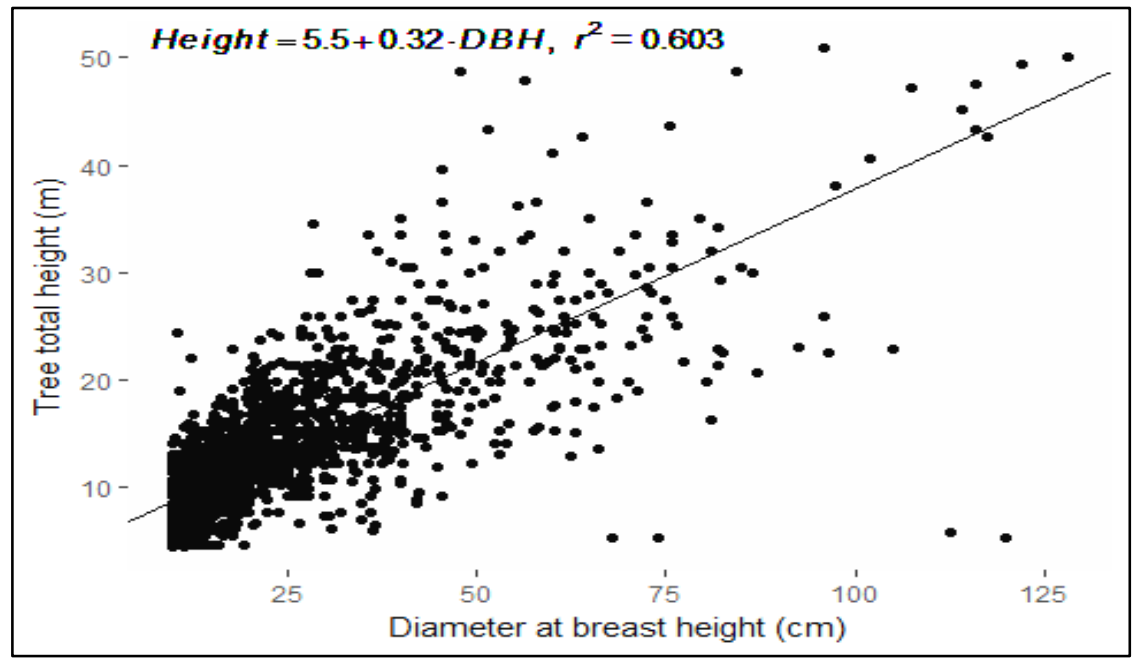

Fig. 3. Height and diameter relationship of the recorded trees in Dudhpukuria-Dhopachari WS.

Bhuju and Yonzon (2009) studied height classes of Shorea robusta, Schima wallichii, Terminalia alata in a dynamic landscape of the Churiya, eastern Nepal, where, all the three species were found in highest frequency within 4-10 m height range. The height class distribution shows existence of a well vertically stratified natural forest in Dudhpukuria-Dhopachori Wildlife Sanctuary. D. turbinatus, S. floribunda, D. alatus, D. costatus, Artocarpus chama, Syzygium firmum, Bombax insigne, Terminalia bellirica dominated the upper canopy. Lithocarpus acuminata, Ficus variegata, Elaeocarpus floribundus, Dillenia pentagyna, Artocarpus lacucha, Acronychia pedunculata, Grewia nervosa, Hydnocarpus laurifolius, Macaranga denticulata, Mitragyna parvifolia, Protium serratum, Schima 
wallichii, Stereospermum colais, Tarenna campaniflora, Vitex peduncularis appeared as the predominant tree species in the study. The forest of this region is known as "Garjan (Bangali name of Dipterocarpus spp.) forest" because, Dipterocarpus spp. were most dominant in this forest. Severe deforestation, over-exploitation and human settlement at the adjacent forest areas in the past decades caused population decrease of dominant trees in the upper canopy. But, after declaring the area as Wildlife Sanctuary, protection status of this forest is enhanced and co-management programs are in operation with the local people. Extraction of forest resources is in a reducing trend. The results of the study suggest continuation of protection activities so that a better stratified natural forest development is ensured with proficient tree population in each stratum.

The tree species richness, density, population structure and recruitment of trees in DudhpukuriaDhopachori Wildlife Sanctuary are far richer than the other natural forests of Bangladesh. The regeneration status of the trees could be further enhanced by strengthening the present protection measures and awareness. Findings of the present study may help the authority of the protected area in prioritizing species specific conservation programs and adopting selective system of timber extraction (if any in future) from the Dudhpukuria-Dhopachori Wildlife Sanctuary.

\section{ACKNOWLEDGEMENTS}

The financial support provided by Arannayk Foundation as well as permission and support of Forest Department during field work is greatly acknowledged by the authors.

\section{REFERENCES}

Ahmed, G. U. and S. M. S. Haque. 1993. Percentage distribution of species and diameter class in natural forests of Bangladesh. The Chittagong University Studies, Part II. 17(1): 109-113.

Alam, M. K. 2008. Forests and Forestry in Bangladesh. In: Z. U. Ahmed, Z. N. T. Begum, M. A. Hassan, M. Khondker, S. M. H. Kabir, M. Ahmad, A. T. A. Ahmed, A. K. A. Rahman, and E. U. Haque (eds.). Encyclopedia of Flora and Fauna of Bangladesh. Vol. 1. Bangladesh Profile. Asiatic Society of Bangladesh, Dhaka., pp. 73-86.

Altrell, D., M. Saket, L. Lyckebäck and M. Piazza. 2007. National Forest and Tree Resource Assessment 2005-2007, Bangladesh. Food and Agriculture Organization (FAO) of the United Nations, Italy and Ministry of Environment and Forest (MoEF), Bangaldesh., pp. 37-40.

Bhuju, D. and P. Yonzon. 2009. Species maintenance in dynamic landscape of the Churiya, Eastern Nepal. The Nature Conservation Society of Japan. 10: 1-10.

Campbell, D. G., D. C. Daly, G. T. Prance and U. N. Maciel. 1986. Quantitative ecological inventory of Terra Firm and Varzea tropical forest on the Rio-Xingu Brazilian Amazon. Brittonia. 38: 369-93.

Das, D. K. and M. K. Alam. 2001. Trees of Bangladesh. Bangladesh Forest Research Institute, Chittagong., pp. 1-10.

Das, S. 1980. Dipterocarp forests of Bangladesh and their management. Bono Biggyan Patrika. 9(1 and 2): 71-86.

Gentry, A. H. 1988. Changes in plant community diversity and floristic composition on environmental and geographic gradients. Annals of the Missouri Botanical Garden. 75: 1-34. 
Haque, S. M. S. and M. S. Alam. 1988. Some aspects of practicing the clear felling followed by artificial regeneration system in the Cox's Bazar Forest Division. Chittagong University Studies, Part II. 12(2): 87-95.

Hassan, M. M. 1995. Biodiversity Conservation and Management in Bangladesh: A state of the Art. International Centre for Integrated Mountain Development (ICIMOD), Katmandu, Nepal.

Hossain, M. A., M. K. Hossain, M. Shafiul Alam and M. Kalimuddin Bhuiyan. 2014. Floristic composition of a Protected Area of Dudpukuria-Dhopachari Wildlife Sanctuary, Chittagong, Bangladesh. Bangladesh Agriculture. 6(1): 38-70.

Khan, M. L., J. P. N. Rai and R. S. Tripathi. 1987. Population structure of some tree species in disturbed and protected subtropical forests of northeast India. Acta Oecologia. 8: 247-255.

Kimmins, J. P. 1996. Forest Ecology: A Foundation for Sustainable Management. 2nd ed. Prentice hall, USA. $611 \mathrm{pp}$.

Kwit, C. and W. J. Platt. 2003. Disturbance history influences regeneration of non- pioneer under storey trees. Ecology. 84(10): 2575-2578.

Lammerts van Bueren, E. M. and J. F. Duivenvoorden. 1996. Towards priorities of biodiversity research in support of policy and management of tropical rain forests. The Tropenbos Foundation, Wageningen, Netherlands. 35 pp.

Motaleb, M. A. and M. K. Hossain. 2009. Studies on the structural composition based on diameter class distribution of a semi evergreen natural forest of Chittagong (South) Forest Division, Bangladesh. Eco-friendly Agril. J. 2(10): 825-829.

Nanos, N. and G. Montero. 2002. Spatial prediction of diameter distribution models. Forest Ecol. Manag. 161(1): 147-158.

Nath, T. K., M. K. Hossain and M. K. Alam. 1997. Studies on the structural composition of a natural forest of Chittagong Hill Tracts (South) Forest Division based on diameter class distribution. The Chittagong University Studies, Part II: Science. 21(1): 15-22.

Newton, P. F. and V. G. Smith. 1988. Diameter distributional trends within mixed Black-spruce/Balsamfir and pure Black spruce stand types. Forest Ecol. Manag. 25: 123-138.

Rankin-de-Merona, J. M., G. T. Prance, R. W. Hutching, M. F. Silva, W. A. Rodrigues and M. E. Uehling. 1992. Preliminary results of a large-scale tree inventory of upland rain forest in the central Amzon. Acta Amazonica. 2: 493-534.

SBSTTA. 1996. Biological diversity in forests. Note by the Secretariat. Convention on Biological Diversity. UNEP/CBD/SBSTTA/2/11.

Shaforth, P. B, J. C. Stromberg and D. T. Patten. 2002. Riparian vegetation response to altered disturbance and stress regimes. Ecological Applications. 12: 107-123.

Sheykholeslami, A., K. H. Pasha and K. A. Lashaki. 2011. A study of tree distribution in diameter classes in natural forests of Iran (Case Study: Liresara Forest). Ann. Biol. Res. 2(5): 283-290. 